\title{
Internet of Things Based Wireless Plant Sensor for Smart Farming
}

\author{
M. Monica Subashini ${ }^{1 *}$, Sreethul Das ${ }^{2}$, Soumil Heble ${ }^{3}$, Utkarsh Raj ${ }^{4}$, R Karthik ${ }^{5}$ \\ ${ }^{1,3,4}$ School of Electrical Engineering, VIT University, Vellore, Tamilnadu, India \\ ${ }^{2}$ School of Mechanical Engineering, VIT University, Vellore, Tamilnadu, India \\ ${ }^{5}$ Department of Electronics and Communication Engineering, MLR Institute of Technology, Dundigal, Hyderabad, India
}

\begin{tabular}{l} 
Article Info \\
\hline Article history: \\
Received Nov 16, 2017 \\
Revised Jan 23, 2018 \\
Accepted Feb 18, 2018 \\
\hline
\end{tabular}

\section{Keywords:}

ESP8266

IoT

Smart farming

Thingspeak

Wi-Fi

\begin{abstract}
About $10 \%$ of the world's workforce is directly dependent on agriculture for income and about $99 \%$ of food consumed by humans comes from farming. Agriculture is highly climate dependent and with global warming and rapidly changing weather it has become necessary to closely monitor the environment of growing crops for maximizing output as well as increasing food security while minimizing resource usage. In this study, we developed a low cost system which will monitor the temperature, humidity, light intensity and soil moisture of crops and send it to an online server for storage and analysis, based on this data the system can control actuators to control the growth parameters. The three tier system architecture consists of sensors and actuators on the lower level followed by an 8-bit AVR microcontroller which is used for data acquisition and processing topped by an ESP8266 Wi-Fi module which communicates with the internet server. The system uses relay to control actuators such as pumps to irrigate the fields; online weather data is used to optimize the irrigation cycles. The prototyped system was subject to several tests, the experimental results express the systems reliability and accuracy which accentuate its feasibility in real-world applications.
\end{abstract}

\section{Corresponding Author:}

M Monica Subashini,

School of Electrical Engineering, VIT University,

Vellore, Tamilnadu, India

Email: monicasubashini.m@vit.ac.in

\section{INTRODUCTION}

Agriculture is the science and an art of cultivating crops for the purpose of economic gain or consumption. India ranks second in global farm output and about 17\% of the GDP of India is accredited to agriculture and its allied sectors, which employ about half of the country's workforce [1].

Agriculture plays a cardinal role in human existence and development, but it is not yet in sync with today's IT age. Climate change due to global warming, along with rapid population growth has put a lot of burden on the agriculture sector to provide food and other products for consumption and the traditional farming practices are striving to satisfy the demands [2].

Every crop has its own comfortable environment for growth and it is necessary to monitor it because the environment of a crop plays a major role in its quality, yield and also in the spread of diseases [3]. Manual inspection of the crop status can be spotty, error prone and can be labour intensive for large fields [4].

The use of sensor networks to constantly monitor the growth parameters of crops such as soil moisture, temperature, relative humidity, etc. give farmers valuable data to allow better control of resources, improve quality and yield of crops, quickly respond to adverse growing conditions, automatic control of farm 
equipment, cut labour costs among other benefits. This technology along with the use of the internet can provide ever better functionality and understanding of the crop growth dynamics [5].

The collected data can be uploaded to a server to be used as a rich data set for agricultural research or it can be integrated along with other available online data such as crop databases, weather databases, etc. to aid the farmers with regional or condition specific tips related to agriculture.

Communication framework in farm monitoring systems can be either wired or wireless, but the advantages of wireless systems outclass those of wired systems. Some of the examples of wired communication systems used are RS485 and CANBUS [6]. Short range wireless communication system examples include Zigbee, Bluetooth, RF, Wi-Fi and long range wireless communication systems are comprised of GSM and GPRS [7].

Wired systems are costly and consume a lot of power due to extensive wiring requirements, these systems are neither flexible nor easily scalable and the whole system needs restructuring when new systems are added [8-9]. Wireless systems are low cost, low power, easily expansible and reconfigurable.

Existing wireless farm monitoring systems use a gateway to connect to the internet and use components which may be costly to produce the final product and for a product to be widely used, it needs to be low cost and reliable [10]-[12].

The developed prototype uses low-cost, stable sensors along with a low-cost Wi-Fi module and microcontroller. In addition to sensor data logging and display the prototype uses local weather data to optimize irrigation cycles to efficiently use natural resources and avoid flooding of fields.

\section{BACKGROUND}

There is a lot of research going on in modernizing agriculture practices, new instruments and techniques are being developed to tackle critical problems related to resource utilization, crop security, yield improvement etc.

Basic logging systems were developed by researchers in 2010, which monitored the crop's temperature and water status and stored the time stamped data in an EEPROM for retrieval at a later date. The system only monitored a few environmental parameters and its field deployment life was limited by the EEPROM size, once the space ran out the user has to retrieve the data so that new data can be logged [13].

Advancements in wireless communication and sensor technologies allowed researchers to add better functionality to smart farming systems. A smart wireless sensor network was designed by researchers for agricultural environment in 2011 which uses Wi-Fi communication to transmit sensor data to a local server for logging and storage, the system uses deep sleep modes and low power sensors for longer battery life [4].

As internet access became cheaper and widespread researchers exploited its potential benefits by pushing the acquired sensor network data online for storage, analytics and easy access. In 2013, researchers developed a wireless sensor network based on Zigbee protocol and used an internet gateway to transfer the collected data from all the nodes to an online database [14]. The system also allowed farmers to control the field's irrigation by logging in to a dedicated web account.

A paper published in 2014 studies the advantages of using weather forecast data to make strategic decisions in farming practices and the economic benefit it brings [15]. Another paper studied the integration of weather forecast data into farming practices and has shown a significant improvement in crop quality and yield [16]. Several models have also been researched and developed for pest management which are based on weather information. Since pests are season and weather dependent, the use of predictive models and weather information allow efficient use of pesticides [17].

Precision agriculture came into play when people started integration spatial and temporal farming data. The proliferation of web based services enabled people to use public databases to their advantage. The 2014 research paper outlines a method which controls farming operations based on spatial data obtained from various web services [18]. The research used web map service (WMS) and web feature service (WFS) to acquire site-specific data such as weather and forecast data, ground water data etc. to plan the control of various farming activities such as irrigation and pesticide spraying. The research has exhibited a solution to use geo-spatial data from multiple sources to control farm activities and enhance crop growth.

\section{METHODOLOGY AND SYSTEM ARCHITECTURE}

The prototype architecture is composed of 5 modules, namely the power supply system, sensor system, output and actuation system, control system and the internet communication system as shown in Figure 1. 


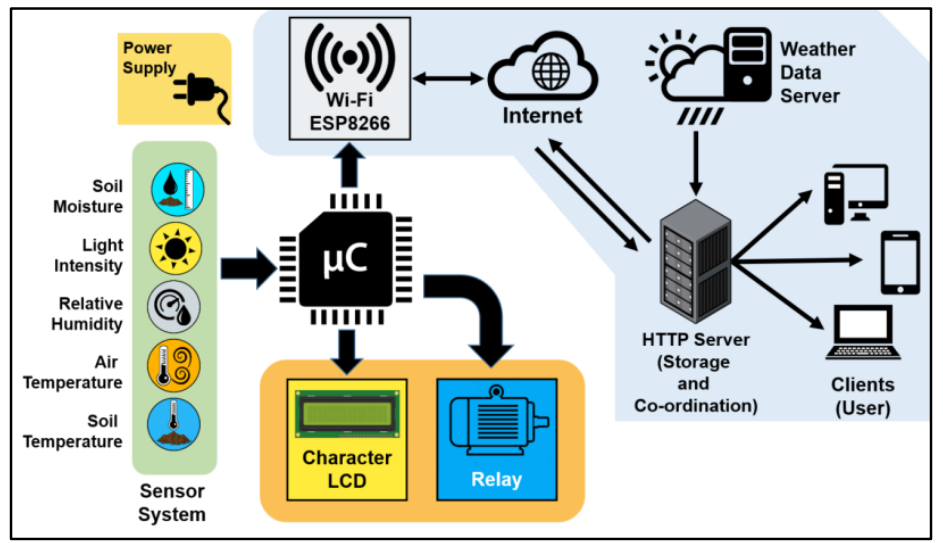

Figure 1. System Architecture

The power supply system manages the task of distributing required power to the components and regulating the voltage from the battery. The majority of components used are capable of running at $5 \mathrm{~V}$ hence a $5000 \mathrm{mAh} \mathrm{Li}$-ion battery capable of supplying $5 \mathrm{~V}$ at $2.1 \mathrm{~A}$ is used to power the device. Additionally to supply 3.3V to the ESP8266 Wi-Fi module, AMS1117 (Advanced Monolithic Systems) low dropout voltage regulator is used.

The sensor system consists of five different sensors measuring the crop growth environment for soil moisture, light intensity, relative humidity, air temperature and soil temperature. The sensors used are lowcost, commercial off the shelf solutions which are known to produce reliable measurements. The required signal conditioning circuits are designed for the sensors such that they produce a response in suitable form. The sensors used are thermistor for soil temperature measurement, co-planar capacitor for soil moisture measurement, photo-resistor for light intensity measurement and DHT11 for air temperature and relative humidity measurement.

Output and actuation system comprises a 16x2 character LCD (JHD162A) based on the Hitachi HD44780 LCD controller to display the measured values and a relay module, used for controlling the irrigation pump based on soil moisture levels and weather forecast data from the internet.

The internet communication system is made up of ESP8266 ESP-12 Wi-Fi module which is the system's gateway to the internet. The collected data is sent to the Wi-Fi module via UART which then pushes the data to the ThingSpeak HTTP server using GET requests. The ThingSpeak platform, then plots the data on a time chart which the user can access by logging into the account. The ThingSpeak server also coordinates with a weather server via HTTP GET requests to send weather forecast data to the system which is then utilized for optimizing irrigation cycles.

The control system is basically the microcontroller which co-ordinates all the other systems. The microcontroller used is Atmel's high-performance ATMega328 microcontroller which is an 8-bit RISC architecture microcontroller. It is responsible for measuring sensor data, displaying measured data onto the $\mathrm{LCD}$, sending sensor data to the $\mathrm{Wi}-\mathrm{Fi}$ module in the correct format, requesting weather forecast data from the server via the Wi-Fi module and then controlling the relay based on soil moisture readings and the forecast data.

The following design specifications were considered while developing the system:

a. Use of reliable, low-cost sensors for monitoring

b. Monitoring environmental parameters having significant impact on the crop growth

c. Incorporating aspects of IoT and wireless sensor networks by wirelessly transmitting data to an online server and using weather data to optimize irrigation cycles.

d. Inclusion of basic control of farm equipment such as a water pump by using relays

e. Using high grade components and keeping bill of materials as low as possible.

\section{SENSOR INTEGRATION AND SIGNAL CONDITIONING}

\subsection{Thermistor}

A thermistor is a two terminal, non-polar device made up of semiconductor material which is usually used for the purpose of temperature measurement. A thermistor having negative temperature coefficient (NTC) with a nominal resistance of $10 \mathrm{k} \Omega$ is being used for measuring the soil temperature. An NTC 
thermistor reduces its resistance as its temperature rises. Thermistors are economical and accurate, requiring a minimal amount of extra circuitry for interfacing [19-20].

The thermistor forms a voltage divider along with a $10 \mathrm{k} \Omega$ resistor and the voltage at the junction is measured by the microcontroller via an ADC, refer Figure 2.

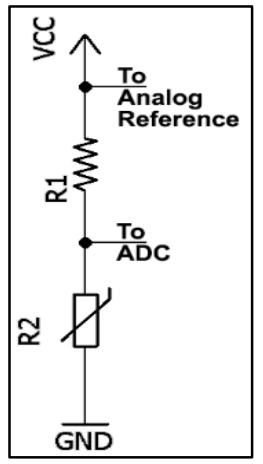

Figure 2. Thermistor Microcontroller Interfacing

The voltage measured depends upon the resistance of the thermistor which is a function of its temperature. The ADC value is sampled five times and averaged and then the thermistor's resistance is extracted using the formula:

$$
R_{T}=\frac{R_{1}}{\left(\frac{1023}{A D C_{-} V A L U E}-1\right)}
$$

Upon obtaining the resistance value, the thermistor temperature is calculated using the $\beta$ parameter equation, defined as:

$$
\frac{1}{T}=\frac{1}{T_{0}}+\frac{1}{\beta} \ln \left(\frac{R}{R_{0}}\right)
$$

Where $\mathrm{T}=$ Measured Temperature (in $\mathrm{K}$ ), $\mathrm{T} 0=$ Nominal Temperature $(298 \mathrm{~K}), \beta=\beta$ value of thermistor (3977), $\mathrm{R}=$ thermistor resistance, $\mathrm{R} 0=$ Resistance of thermistor $(10 \mathrm{k} \Omega)$ at nominal temperature. The thermistor $\beta$ value, T0 and R0 is obtained from the manufacturer's datasheet. The R0 value was verified using Pt100 RTD and the updated value was used for calculation of the temperature.

\subsection{Light Intensity Sensor}

A photocell, photo-resistor or light dependent resistor (LDR) is a non-polar, two terminal device whose resistance depends upon the light intensity incident on it. A calibrated CdS photocell is used for measuring the incident light intensity. LDRs are cheap, long lasting, have high stability and consume low current in the dark [21]. The dark resistance of a photocell is in the order of mega-ohms (M $\Omega$ ) and incident light can bring it down to the order of several hundred ohms. The above mentioned voltage divider setup is used to measure the resistance of the photocell, refer Figure 3.

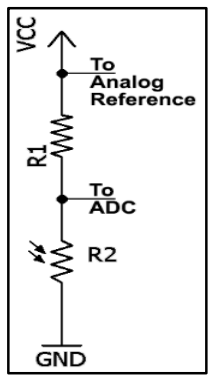

Figure 3. LDR Microcontroller Interfacing 
The resistance of a photocell exponentially falls with increasing light intensity, hence the pull up resistor needs to be selected based on the required region of light sensitivity. Since we are interested in measuring the light intensity during the daytime a pull up resistor of $1 \mathrm{k} \Omega$ was used. The photocell was calibrated by comparing its resistance values with the light intensity value (in Lux) given by Liteon LTR303ALS-01 optical sensor. The photocell and Liteon sensor were exposed to light of varying intensity produced by six warm white LED driven by PWM. The setup of the calibration is shown in the Figure 4.

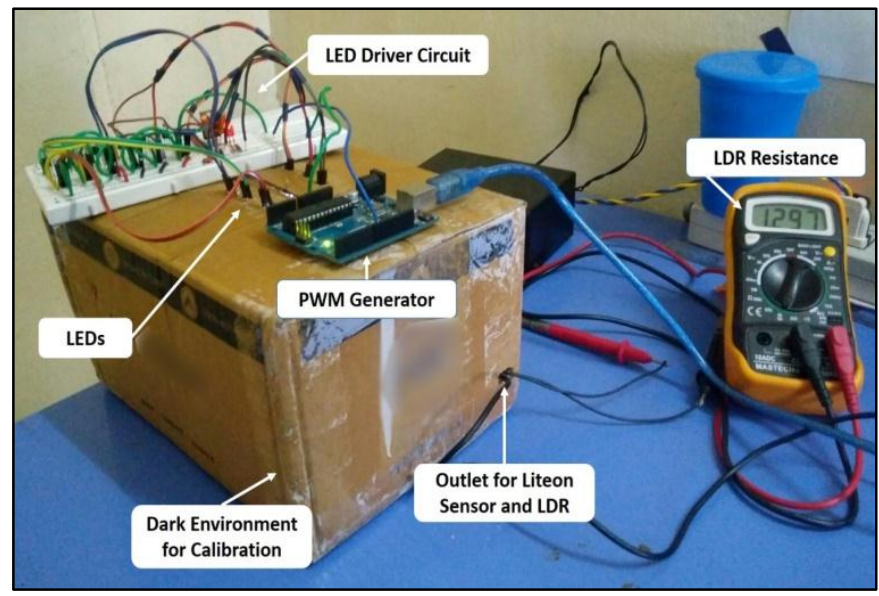

Figure 4. LDR Calibration Setup

The recorded values were fed to MATLAB and power law curve fitting technique was used to obtain a formula for converting the photocell resistance to light intensity (in Lux), refer Figure 5.

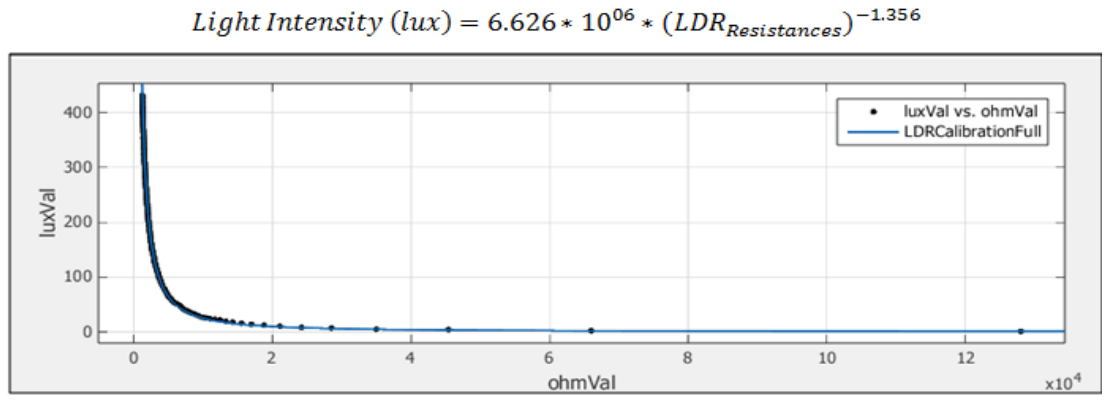

Figure 5. LDR Calibration/Curve Fitting Tool

\subsection{Relative Humidity Sensor}

DHT11 is a low-cost, composite, pre-calibrated, digital temperature and relative humidity sensor. The sensor package consists of a resistive humidity sensor and an NTC thermistor for temperature measurement also a high performance 8-bit microcontroller with on-board ADC for measurement and conversion of sensors signal. The sensor module can be interfaced with microcontroller by communicating over its proprietary single wire communication interface, refer [22]. The microcontroller provides the humidity and temperature data as 8-bit integer and 8-bit decimal format with an 8-bit CRC for ensuring reliable data reception, refer figure 6 for the communication frame. The module has a measurement range of $20-90 \%$ relative humidity and $0-50{ }^{\circ} \mathrm{C}$ with an accuracy of $\pm 5 \% \mathrm{RH}$ and $\pm 2{ }^{\circ} \mathrm{C}$ respectively. 


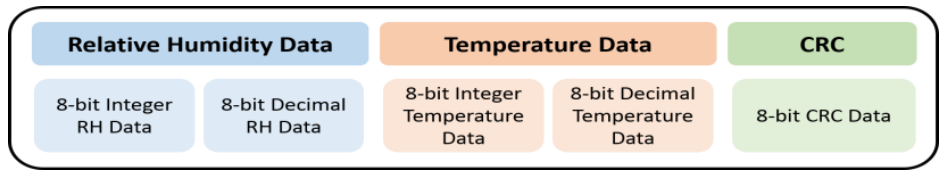

Figure 6. DHT11 Communication Data Frame

The resistive humidity sensor is preferred because of its low-cost, high accuracy, compact size and near linear voltage output [20]. The sensor module is connected to the microcontroller using a $1 \mathrm{k} \Omega$ pull-up resistor for the one wire communication, refer Figure 7 and Figure 8.

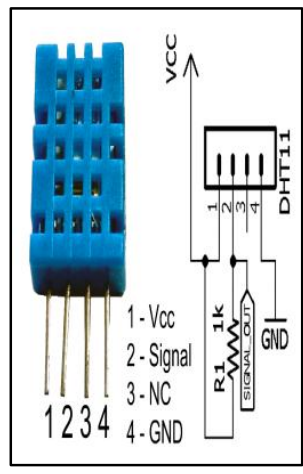

Figure 7. DHT11

Microcontroller Interfacing

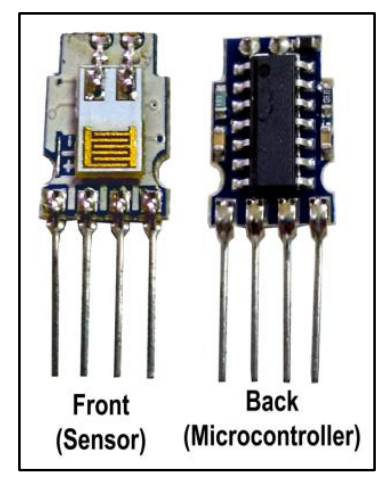

Figure 8. DHT11 Internals

\subsection{Soil Moisture Sensor}

Soil moisture measurement is paramount, because without water plants cannot survive for long and its value acts as a gauge for irrigation control. Many non-destructive soil moisture measurement methods have been developed and used over the years, such as frequency domain reflectometry (FDR), time domain reflectometry (TDR), resistance measurement, dielectric change measurement and many more. FDR and TDR measurement methods, though accurate have a complex design requiring a lot of precision electronics which makes them costly to incorporate [23]. The resistance method, though very cheap to implement, lacks accuracy and is affected by temperature and change in ionic content of the soil requiring frequent calibration [23]. The prototype is equipped with a fringing electric field, capacitive soil moisture sensor which exploits the fact that the dielectric constant of water $(\varepsilon=81)$ is about twenty times that of the soil $(\varepsilon=4)$. Capacitive soil moisture sensors are robust, cheap and perform non-destructive measurement. The sensor consists of two interdigitated electrodes on a PCB and the design is based on the guidelines outlined by the following research papers [24] and [25].

The capacitance of the sensor is converted to voltage signal for measurement by the microcontroller. The capacitive sensor forms a low pass filter, filtering a square wave signal and charging a large capacitor with the rectified output of the filter. The voltage of the larger capacitor is proportional to the capacitance of the sensor which is an indicator of the soil moisture. The signal conditioning system consists of 5 blocks. The first block is a 555 timer based square wave generator producing $100 \mathrm{kHz}$ signal with approximately $50 \%$ duty cycle. The second block is the low pass filter of which the sensor forms a part. The resistor for the filter is chosen based on the square wave frequency and the capacitance change over the measurement range, for the designed sensor it turned out to be around $93 \mathrm{k} \Omega$. Two transistors in the second block discharge the capacitor when the signal is low, producing greater measurement range. Since the capacitance of sensor is very low and prone to loading effects the third block consists of a precision rectifier which is based on LM358 opamp and 1N4148 small signal diode. The rectified signal is fed to the fourth block which is called the charge bucket circuit because it consists of a large capacitor $(1 \mathrm{uF})$ collecting charge generated by the low pass filter, a large resistor is in parallel with the capacitor to provide a discharge path when there is change in the filters cut-off frequency. The final block is a voltage buffer utilizing the second opamp in the LM358 package, refer Figure 9 for the schematic. 


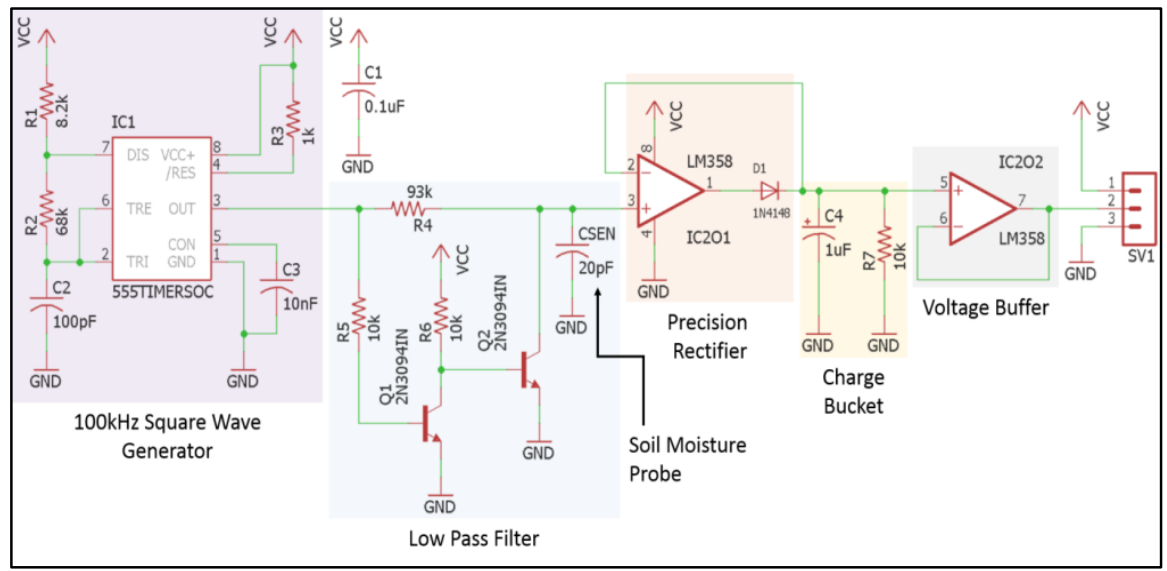

Figure 9. Soil Moisture Sensor Signal Conditioning Circuit

The sensor was calibrated for different values of volumetric water content (ratio of volume of water to the volume of soil). Sun-dried clay was taken in a $700 \mathrm{~mL}$ container in which the sensor was embedded and a measurement was taken. The soil was wetted with $7 \mathrm{~mL}$ of water $(1 \% \mathrm{VWC})$, mixed thoroughly and the voltage from the circuit was measured. This process was repeated till $40 \%$ VWC was reached after which the soil matrix is unable to hold water and the sample is flooded [24], refer Figure 10 for the calibration setup. The readings were fed into MATLAB and piecewise linear functions were generated and used for obtaining the VWC from the measured voltage, refer Figure 11. The calibration test was repeated one more time to ensure the sensor's reliability.

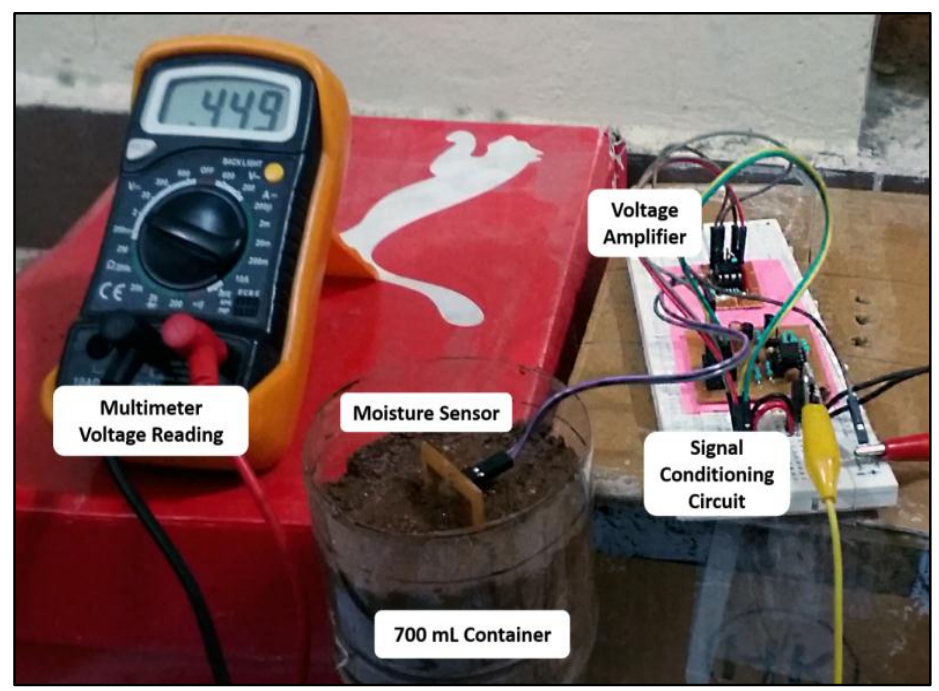

Figure 10. Soil Moisture Sensor Calibration Setup 


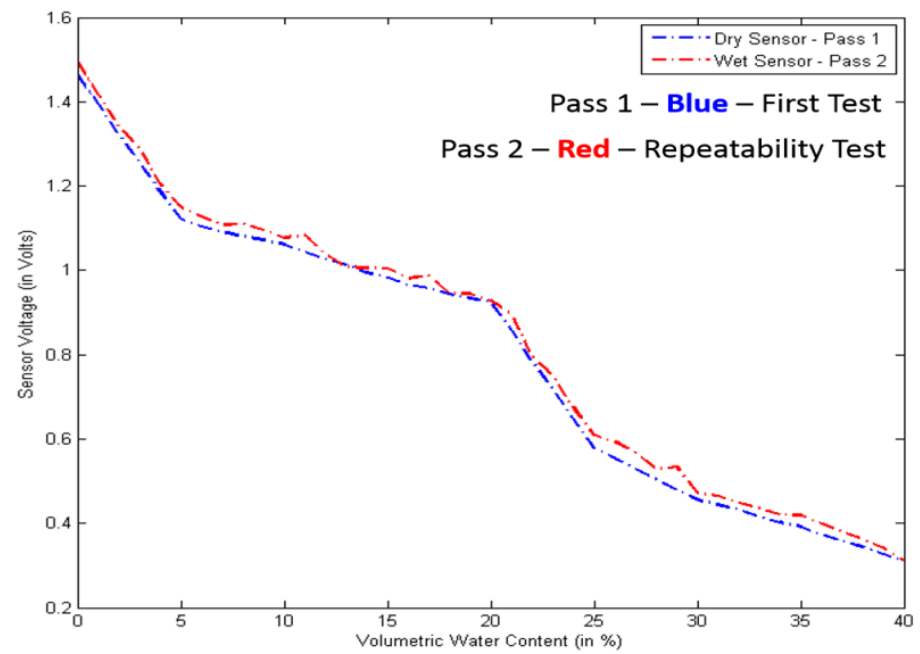

Figure 11. Soil Moisture Sensor Calibration Graph

\section{MICROCONTROLLER AND CLOUD ARCHITECTURE}

\subsection{ESP8266 and Lua Scripts}

The wireless communication over Wi-Fi is handled by ESP8266 ESP-12E Wi-Fi module, which is manufactured by AI-Thinker, refer Figure 12. The ESP8266 ESP-12E is built around the low-cost ESP8266 Wi-Fi SoC. A MOSFET based bi-directional level converter is used for UART communication between the microcontroller running at $5 \mathrm{~V}$ and the ESP8266 running at 3.3V.

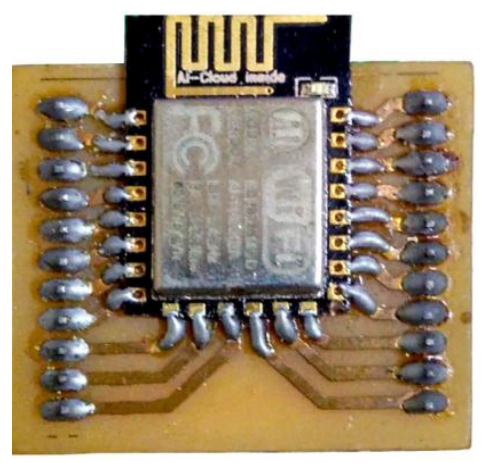

Figure 12. ESP8266 ESP 12E

The ESP8266 was flashed with NodeMCU firmware and programmed to run a Lua script, which on powering up, sets itself to station mode (to connect to the Wi-Fi access point), connects to the Wi-Fi AP (access point) and waits for the 8-bit microcontroller to interrupt, figure 13 describes the program flowchart. The script has 2 hardware interrupts which are triggered by the 8-bit microcontroller when it needs to send data to the server or get weather information from the server, refer Figure 14 for the interrupt routine flowchart. The first interrupt is fired every 20 minutes and the second interrupt is fired only when the soil moisture level falls below the required limit and irrigation is required. 

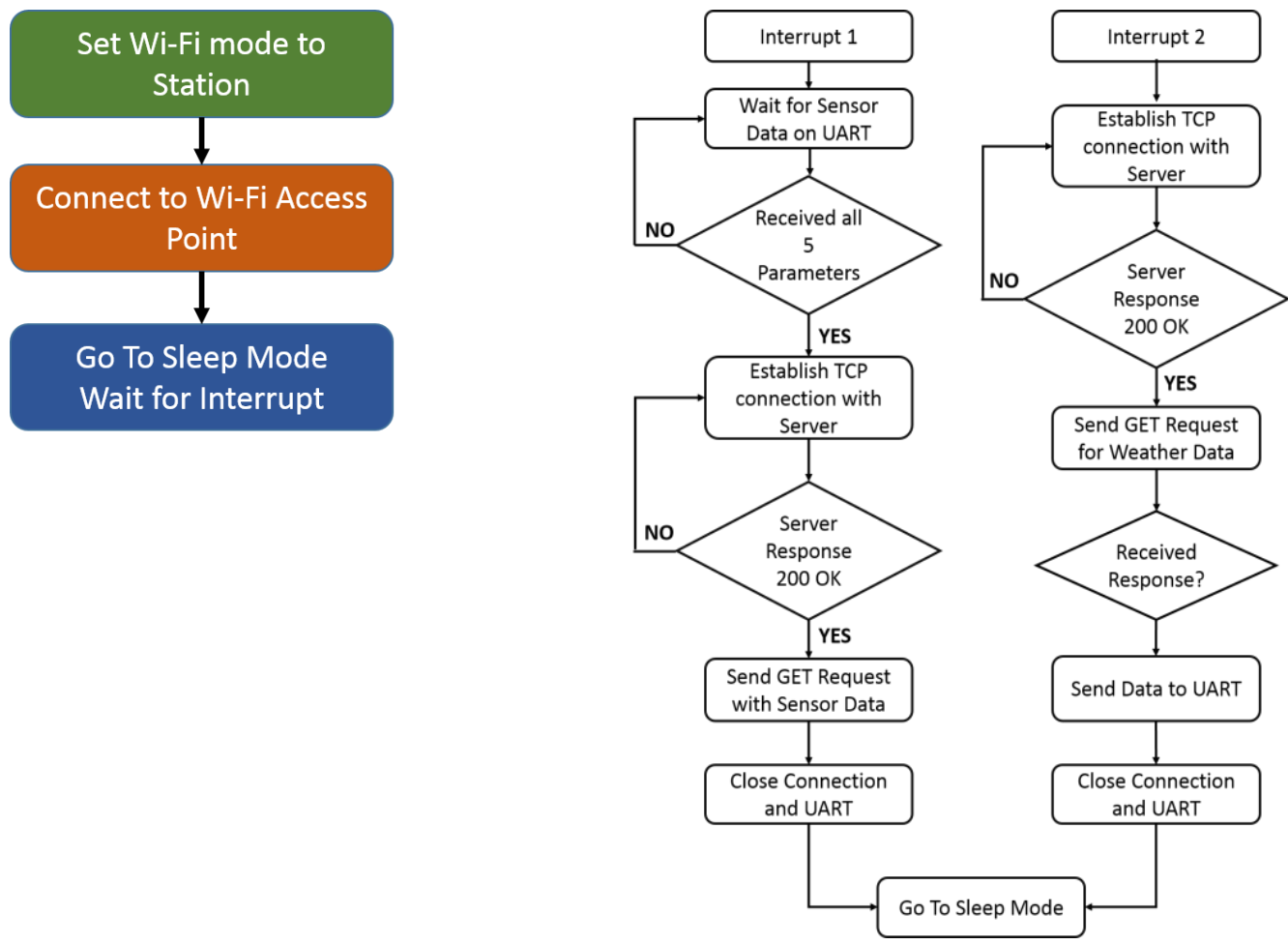

Figure 13. ESP8266 Mail Lua Script

Figure 14. ESP8266 Interrupt Lua Program

\subsection{Weather Underground}

Weather Underground is a San Francisco based company which provides real-time weather updates via the internet. The company also provides access to its API (Application Program Interface) which enables developers to access data from weather underground and use it in their applications. The API key is offered is free of cost, but some features are premium and require paid subscription. The weather underground API provides current weather conditions and weather forecast in JSON or XML format. Users can get their API key by registering on the website and use it to request weather data via HTTP requests.

The following data is extracted from the weather forecast JSON response:

"conditions" - current weather condition

"relative_humidity" - relative humidity (in \%) and

"qpf_allday" - quantitative precipitation forecast (in $\mathrm{mm}$ ), which is the aggregate melted precipitation over a stated period of time in a specified area.

\subsection{ThingSpeak}

ThingSpeak is an open source data platform and API for Internet of Things (IoT) which allows clients or "things" to retrieve and store data using HTTP protocol over the internet. ThingSpeak API and application also offer a rich set of features such as data processing operations like median, averaging etc., time zone management, write/read API key management and much more. The platform allows the creation of channels which support data entry of up to 8 fields, longitude, latitude, channel status and elevation. The channel feeds can be embedded or integrated with other applications as well. Every channel created can be public or private and has a read and write API key for data entry and access.

\subsection{Irrigation Control Algorithm}

The prototype is also programmed to irrigate the fields when the soil moisture falls below a set value, the VWC (Volumetric Water Content) limit. The system, being connected to the internet uses weather forecast data, specifically the quantitative precipitation forecast value of the region to determine whether to irrigate immediately or to wait if precipitation is going to take place in a few hours. A relay is being used to run the pump which is activated based on an algorithm, refer Figure 15 for the irrigation algorithm flowchart. 


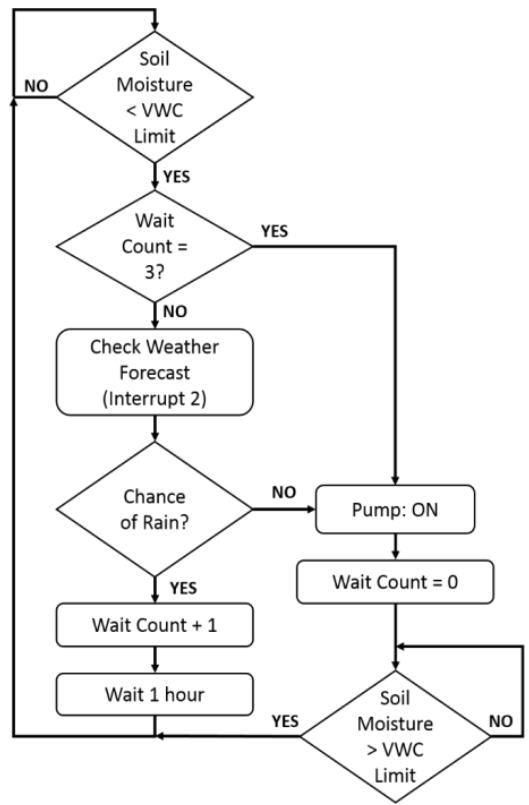

Figure 15. Irrigation Algorithm

\subsection{Master Microcontroller}

The master microcontroller is Atmel's ATMega328 which is chosen because of its low cost and a plethora of features it offers, such as, a large 32kB flash memory, a 10-bit 6-channel ADC, 512 bytes of EEPROM and hardware support for communication protocols such as UART, SPI and I2C. The master microcontroller runs a program which co-ordinates with all the other peripherals in the system. The program flowchart is portrayed in Figure 16.

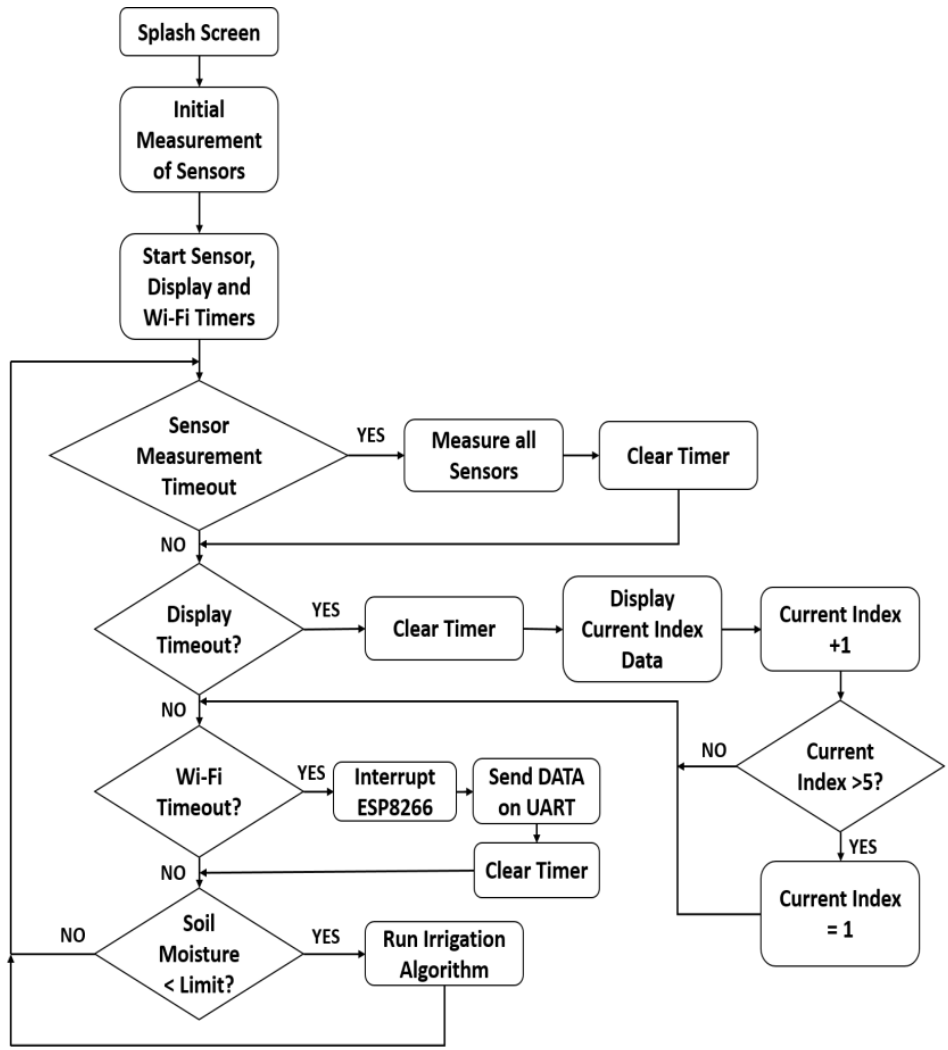

Figure 16. Main Program Flowchart 
The program is entirely interrupt based, a timer is run at the start of the program for sensor measurement, displaying data and for Wi-Fi transmission. When the timer surpasses a set limit the respective routines are triggered and the timers are reset.

\subsection{Field Deployment and Results}

The prototype after completing fabrication was put to test in a controlled environment to validate the sensor accuracy, Figure 17 shows the prototype setup. Temperature sensors for both air and soil temperature were found to be accurate within $\pm 2{ }^{\circ} \mathrm{C}$. The light intensity sensor had a deviation of about \pm 320 lux. The prototype was placed in a pot outdoors under a canopy of trees for 36 hours and the data was updated online every 20 minutes. Figures 18, 19, 20, 21 and 22 show the time plot of the acquired data for air temperature, soil temperature, relative humidity, soil moisture and light intensity respectively.

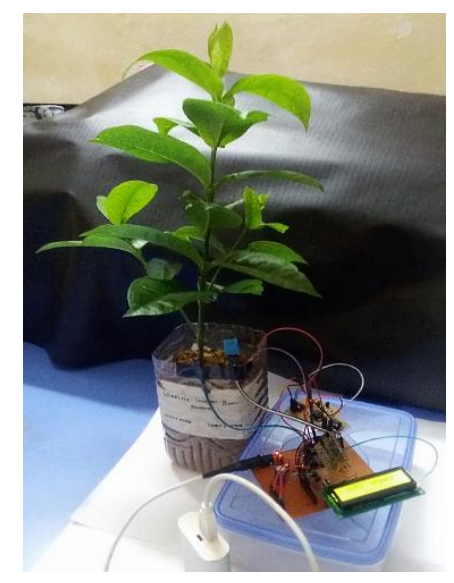

Figure 17. Prototype Setup

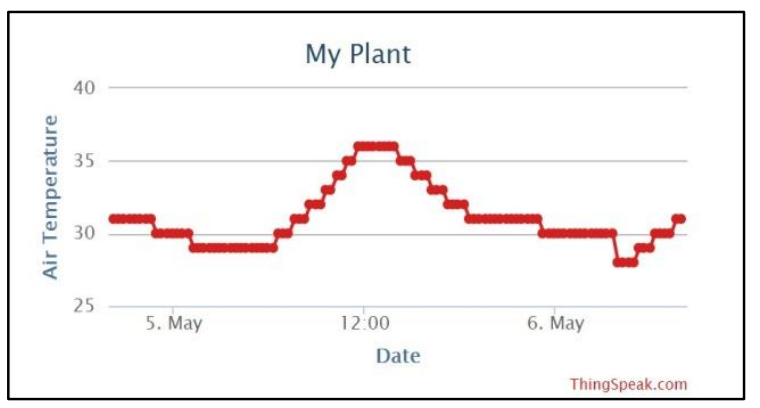

Figure 18. Air Temperature Plot

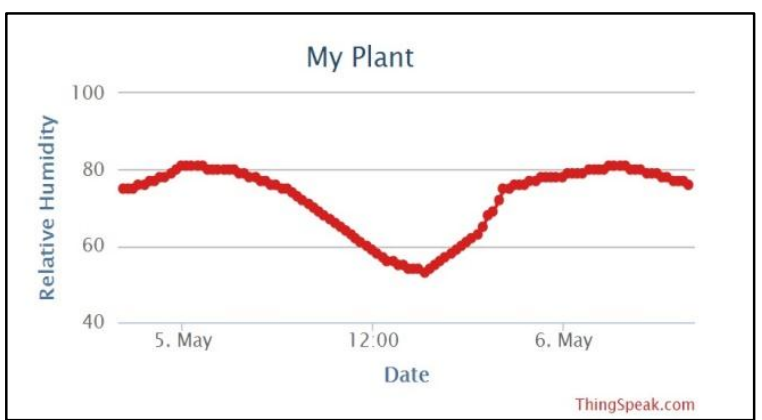

Figure 20. Relative Humidity Plot

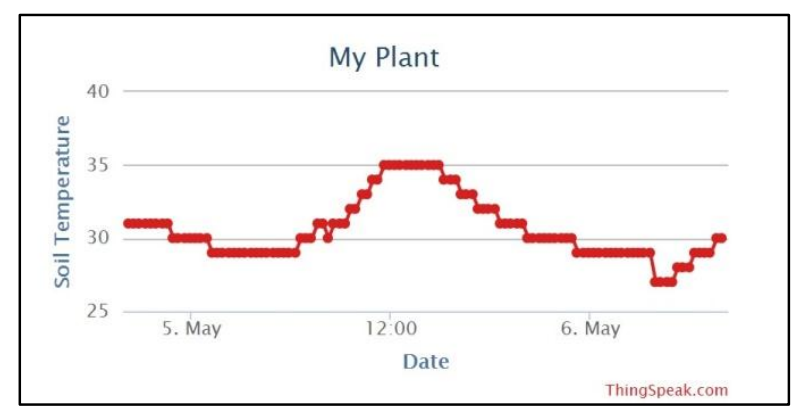

Figure 19. Soil Temperature Plot

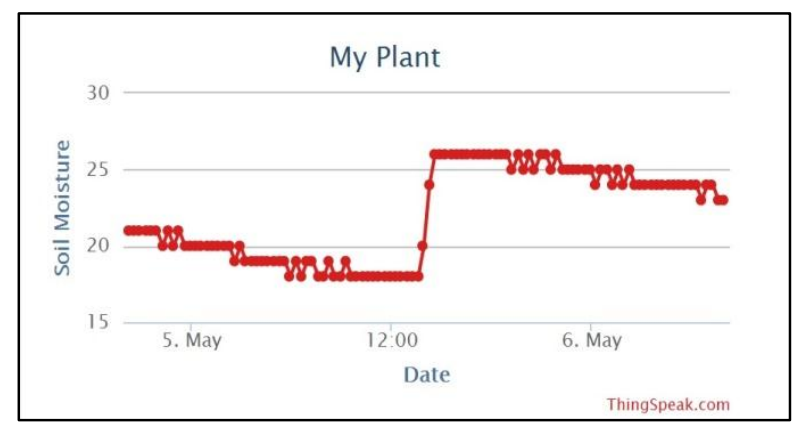

Figure 21. Soil Moisture Plot 


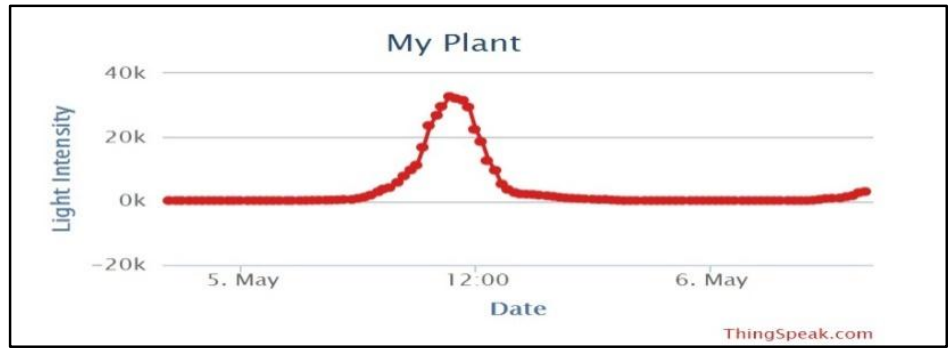

Figure 22. Light Intensity Plot

\section{DISCUSSION AND FUTURE WORK}

The internet of things is picking up pace in the agriculture sector and the introduction of low-cost Wi-Fi solutions have accelerated the development of smart farming systems in agriculture which use the internet and big data analytics to improve efficiency. The developed prototype can be improved further by making it power efficient which involves using sophisticated algorithms to put the wireless module, sensor system and the microcontroller to sleep when any action is not required. The system's portability and life span can be increased by running it entirely on solar power. Its functionality can be increased by developing a Wi-Fi based wireless sensor networks allowing the nodes to communicate with each other, passing data to the internet through the best possible route with least time delay. Moreover, additional functionality such as online control of peripherals, data visualization etc. can be added by to the cloud system by setting up a custom HTTP server and a webpage using Apache 2 Open Source Server and LAMP module in Linux instead if using the ThingSpeak platform.

\section{ACKNOWLEDGEMENTS}

We thank School of Electrical Engineering and School of Mechanical Engineering, VIT University for the support and resources provided for the completion of this project work.

\section{REFERENCES}

[1] M. Datta, C. Neogi, A Sinha, "Sectoral shares in Indian GDP: How to regard it?", Structural Change and Economic Dynamics, Vol. 35, pp.1-11, 2015.

[2] L. Ying, Y Feng, Z. Wei, "Greenhouse environment monitoring system design based on WSN and GPRS networks" IEEE Conference on Cyber Technology in Automation, Control, and Intelligent Systems (CYBER), pp.795-798, 2015.

[3] M. Launay, J. Caubel, G. Bourgeois F. Huard, I. Garcia de Cortazar-Atauri, M. Bancal, N. Brisson, "Climatic indicators for crop infection risk: Application to climate change impacts on five major foliar fungal diseases in Northern France", Agriculture, Ecosystems \& Environment, 197, pp.147-158, 2014.

[4] G. Mendez, M. Md Yunus, S. Mukhopadhyay, "A WiFi based smart wireless sensor network for an agricultural environment”, 2011 Fifth International Conference on Sensing Technology, 2011.

[5] A. Tripathy, J. Adinarayana, D. Sudharsan, S. Merchant, U. Desai, K. Vijayalakshmi, D. Raji Reddy, G. Sreenivas, S. Ninomiya, M. Hirafuji, T. Kiura, K. Tanaka, "Data mining and wireless sensor network for agriculture pest/disease predictions", 2011 World Congress on Information and Communication Technologies, 2011.

[6] L. Guofang, C. Lidong, Q. Yubin, L. Shengtao and X. Junyu, "Remote monitoring system of greenhouse environment based on LabVIEW, " International Conference on Computer Design and Applications, 2010.

[7] V. Singh, I. Singh and S. Sud "Low power embedded controlled sensor network for agricultural applications," IEEE Long Island Systems, Applications and Technology (LISAT) Conference 2014.

[8] Y. Chuanan and Y. Yongchang, "Implementation of greenhouse monitoring system based on RF transceiver," The 2nd International Conference on Industrial Mechatronics and Automation, 2010.

[9] Q, Wang, A. Terzis, and A. Szalay, "A novel soil measuring wireless sensor network," IEEE Instrumentation \& Measurement Technology Conference Proceedings, 2010.

[10] Z. Liao, S. Dai and C. Shen, "Precision agriculture monitoring system based on wireless sensor networks," IET International Conference on Wireless Communications and Applications, ICWCA, 2012.

[11] A. Al-Ali, M. Qasaimeh, M. Al-Mardini, S. Radder and I. Zualkernan, "ZigBee-based irrigation system for home gardens, ” International Conference on Communications, Signal Processing, and their Applications, ICCSPA, 2015.

[12] L. Dan, C. Xin, H. Chongwei and J. Liangliang, "Intelligent Agriculture Greenhouse Environment Monitoring System Based on IOT Technology," International Conference on Intelligent Transportation, Big Data and Smart City, 2015. 
[13] D. Fisher and H. Kebede, "A low-cost microcontroller-based system to monitor crop temperature and water status," Computers and Electronics in Agriculture, 74(1), pp.168-173, 2010.

[14] K. Sathish Kannan and G, Thilagavathi, "Online farming based on embedded systems and wireless sensor networks," International Conference on Computation of Power, Energy, Information and Communication, ICCPEIC, 2013.

[15] P. Calanca, "Weather Forecasting Applications in Agriculture," Encyclopedia of Agriculture and Food Systems, pp.437- 449, 2014.

[16] S. Asseng, P. McIntosh, G. Thomas, E. Ebert and N. Khimashia, "Is a 10-day rainfall forecast of value in dry-land wheat cropping," Agricultural and Forest Meteorology, 216, pp.170-176, 2016.

[17] R. Olatinwo, and G. Hoogenboom, "Weather-based Pest Forecasting for Efficient Crop Protection," Integrated Pest Management, pp.59-78, 2014.

[18] J. Kaivosoja, M. Jackenkroll, R. Linkolehto, M. Weis and R. Gerhards, "Automatic control of farming operations based on spatial web services," Computers and Electronics in Agriculture, 100, pp.110-115, 2014.

[19] J. Kim, and J. Kim, "Voltage divider resistance for high-resolution of the thermistor temperature measurement," 44(10), pp.2054-2059, 2011.

[20] V. Quan, G. Sen Gupta and S. Mukhopadhyay, "Review of sensors for greenhouse climate monitoring," IEEE Sensors Applications Symposium, 2011.

[21] W. Chen, H. Qin and Z. Feng, "Research and design of light intensity detection circuit," International Conference on Electrical and Control Engineering, 2011.

[22] Y. Zhou, Q. Zhou, Q. Kong, and W. Cai, "Wireless temperature \&amp; humidity monitor and control system," 2nd International Conference on Consumer Electronics, Communications and Networks, CECNet, 2012.

[23] Y. Shirahama, R. Shigeta, Y. Kawahara, T. Asami, Y. Kojima, and K. Nishioka, "Implementation of wide range soil moisture profile probe by coplanar plate capacitor on film substrate," IEEE SENSORS, 2015.

[24] R. Dean, A. Rane, M. Baginski, J. Richard, Z. Hartzog and D. Elton, "A Capacitive Fringing Field Sensor Design for Moisture Measurement Based on Printed Circuit Board Technology," IEEE Trans. Instrum. Meas., 61(4), pp.1105-1112, 2012.

[25] J. Mizuguchi, J. Piai, J. de Franca, M. de Morais Franca, K. Yamashita and L. Mathias, "Fringing Field Capacitive Sensor for Measuring Soil Water Content: Design, Manufacture, and Testing," IEEE Trans. Instrum. Meas., 64(1), pp.212-220, 2015.

[26] S Ranjith, Shreyas, K Pradeep Kumar, R Karthik, "Automatic Border Alert System for Fishermen using GPS and GSM techniques", Indonesian Journal of Electrical Engineering and Computer Science (IJEECS), Vol 7, No. 1, (2017). 